\title{
Essential norm of some extensions of the generalized composition operators between $k$ th weighted-type spaces
}

\section{Stevo Stević}

"Correspondence: sstevic@ptt.rs Mathematical Institute of the Serbian Academy of Sciences, Knez Mihailova 36/III, Beograd, 11000, Serbia

Operator Theory and Applications Research Group, Department of Mathematics, King Abdulaziz University, P.O. Box 80203, Jeddah, 21589, Saudi Arabia

\begin{abstract}
We calculate the essential norm of some extensions of the generalized composition operators between $k$ th weighted-type spaces on the unit disk in the complex plane, considerably extending some results in the literature.
\end{abstract}

MSC: Primary 47B38; secondary 47B33; 30H99

Keywords: essential norm; generalized composition operator; kth weighted-type space; unit disk

\section{Introduction}

Let $\mathbb{D}$ be the open unit disk in the complex plane $\mathbb{C}, H(\mathbb{D})$ the class of all holomorphic functions on $\mathbb{D}$, and $S(\mathbb{D})$ the class of all holomorphic self-maps of $\mathbb{D}$.

Let $\mu(z)$ be a positive continuous function on $\mathbb{D}$ (weight) and $k \in \mathbb{N}_{0}$. The $k$ th weightedtype space denoted by $\mathcal{W}_{\mu}^{(k)}(\mathbb{D})=\mathcal{W}_{\mu}^{(k)}$ is defined as follows:

$$
\mathcal{W}_{\mu}^{(k)}=\left\{f \in H(\mathbb{D}): b_{\mathcal{W}_{\mu}^{(k)}}(f)<\infty\right\}
$$

where

$$
b_{\mathcal{W}_{\mu}^{(k)}}(f):=\sup _{z \in \mathbb{D}} \mu(z)\left|f^{(k)}(z)\right|
$$

The space was introduced in [1] where the composition operators from the weighted Bergman space to the space were studied. Some other concrete operators on the space were later studied in [2-4].

If $k=0$, then $b_{\mathcal{W}_{\mu}^{(0)}}(\cdot)$ is a norm on space $\mathcal{W}_{\mu}^{(0)}$, the so-called weighted-type space ([5, 6]). If $k \in \mathbb{N}$, then it is easy to see that $b_{\mathcal{W}_{\mu}^{(k)}}(\cdot)$ is a semi-norm on $\mathcal{W}_{\mu}^{(k)}$. It is not a norm on the space since from $b_{\mathcal{W}_{\mu}^{(k)}}(f)=0$ it follows that $f^{(k)}(z)=0, z \in \mathbb{D}$, and consequently $f(z)=p_{k-1}(z)$, where $p_{k-1}$ is a polynomial of degree at most $k-1$. However, it is a norm on the quotient space $\mathcal{W}_{\mu}^{(k)} / \mathbb{P}_{k-1}$, where $\mathbb{P}_{k-1}$ is the space of all polynomials of degree less than or equal $k-1$. Indeed, let $f+\mathbb{P}_{k-1} \in \mathcal{W}_{\mu}^{(k)} / \mathbb{P}_{k-1}$, and, based on the definition of a norm on

(c) The Author(s) 2017. This article is distributed under the terms of the Creative Commons Attribution 4.0 International License (http://creativecommons.org/licenses/by/4.0/), which permits unrestricted use, distribution, and reproduction in any medium, provided you give appropriate credit to the original author(s) and the source, provide a link to the Creative Commons license, and indicate if changes were made. 
a quotient space, let

$$
\left\|f+\mathbb{P}_{k-1}\right\|_{\mathcal{W}_{\mu}^{(k)} / \mathbb{P}_{k-1}}:=\inf _{g \in f+\mathbb{P}_{k-1}} b_{\mathcal{W}_{\mu}^{(k)}}(g) .
$$

Then, if $\left\|f+\mathbb{P}_{k-1}\right\|_{\mathcal{W}_{\mu}^{(k)} / \mathbb{P}_{k-1}}=0$, by using (1) and (2), we have

$$
0=\inf _{g \in f+\mathbb{P}_{k-1}} b_{\mathcal{W}_{\mu}^{(k)}}(g)=\inf _{p_{k-1} \in \mathbb{P}_{k-1}} b_{\mathcal{W}_{\mu}^{(k)}}\left(f+p_{k-1}\right)_{\mathcal{W}_{\mu}^{(k)}}=b_{\mathcal{W}_{\mu}^{(k)}}(f),
$$

from which it follows that $f \in \mathbb{P}_{k-1}$, that is, $f+\mathbb{P}_{k-1}=\mathbb{P}_{k-1}=0_{\mathcal{W}_{\mu}^{(k)} / \mathbb{P}_{k-1}}$.

On the other hand, there are some natural algebraic isomorphisms between some quotient spaces and some spaces of holomorphic functions. Namely, we have

$$
H(\mathbb{D}) / \mathbb{P}_{k-1} \cong_{a}\left\{f \in H(\mathbb{D}): f^{(j)}(0)=0, j=\overline{0, k-1}\right\}=: H_{k}(\mathbb{D}),
$$

and

$$
\mathcal{W}_{\mu}^{(k)} / \mathbb{P}_{k-1} \cong\left\{\left\{f \in \mathcal{W}_{\mu}^{(k)}: f^{(j)}(0)=0, j=\overline{0, k-1}\right\}=: \mathcal{W}_{\mu, k}^{(k)}(\mathbb{D}) .\right.
$$

Indeed, for each class $g+\mathbb{P}_{k-1} \in H(\mathbb{D}) / \mathbb{P}_{k-1}$ (or $g+\mathbb{P}_{k-1} \in \mathcal{W}_{\mu}^{(k)} / \mathbb{P}_{k-1}$ ) there is a unique $f_{g} \in g+\mathbb{P}_{k-1}$ such that $f_{g}^{(j)}(0)=0, j=\overline{0, k-1}$. Namely, if $g(z)=\sum_{j=0}^{\infty} a_{j} z^{j}$, then we can take $f_{g}(z)=\sum_{j=k}^{\infty} a_{j} z^{j}$, that is, $f_{g}=g+p_{g, k-1}$, where $p_{g, k-1}(z)=\sum_{j=0}^{k-1}\left(-a_{j}\right) z^{j}$, and the map

$$
L\left(g+\mathbb{P}_{k-1}\right):=f_{g}
$$

is a linear bijection from $H(\mathbb{D}) / \mathbb{P}_{k-1}$ onto $H_{k}(\mathbb{D})$, as well as from $\mathcal{W}_{\mu}^{(k)} / \mathbb{P}_{k-1}$ onto $\mathcal{W}_{\mu, k}^{(k)}(\mathbb{D})$. Hence, we can identify the quotient spaces with the corresponding subspaces of holomorphic functions satisfying the conditions $f^{(j)}(0)=0, j=\overline{0, k-1}$.

From (1) and (2) it follows that

$$
\left\|f+\mathbb{P}_{k-1}\right\|_{\mathcal{W}_{\mu}^{(k)} / \mathbb{P}_{k-1}}=b_{\mathcal{W}_{\mu}^{(k)}}(f),
$$

this fact along with the above mentioned algebraic isomorphism shows that the spaces $\left(\mathcal{W}_{\mu}^{(k)} / \mathbb{P}_{k-1},\|\cdot\|_{\mathcal{W}_{\mu}^{(k)} / \mathbb{P}_{k-1}}\right)$ and $\left(\mathcal{W}_{\mu, k}^{(k)}(\mathbb{D}), b_{\mathcal{W}_{\mu}^{(k)}}(\cdot)\right)$ are isometrically isomorphic, that is, $\mathcal{W}_{\mu}^{(k)} / \mathbb{P}_{k-1} \cong \mathcal{W}_{\mu, k}^{(k)}(\mathbb{D})$. So, they can be identified, and we can regard it to be the same if we say $f \in \mathcal{W}_{\mu}^{(k)} / \mathbb{P}_{k-1}$ or $f \in \mathcal{W}_{\mu, k}^{(k)}$.

Let

$$
\|f\|_{\mathcal{W}_{\mu}^{(k)}}=\sum_{j=0}^{k-1}\left|f^{(j)}(0)\right|+\sup _{z \in \mathbb{D}} \mu(z)\left|f^{(k)}(z)\right|,
$$

where $\mu$ is a weight and $k \in \mathbb{N}_{0}$ (for $k=0$ we use the standard convention $\sum_{j=l}^{l-1} a_{j}=0$, $l \in \mathbb{Z})$. Then it is easy to see that (3) defines a norm on space $\mathcal{W}_{\mu}^{(k)}$, and that $\left(\mathcal{W}_{\mu}^{(k)},\|\cdot\|_{\mathcal{W}_{\mu}^{(k)}}\right)$ is a Banach space. The normed space is a natural generalization of the weighted-type, Bloch-type and Zygmund-type spaces (see, e.g., [7-10]).

Let $L: X \rightarrow Y$ be a linear bounded operator, that is, it maps bounded sets of $X$ into bounded sets of $Y$. By $\|L\|_{X \rightarrow Y}$, we denote the operator norm of $L: X \rightarrow Y$, that is, 
$\|L\|_{X \rightarrow Y}=\sup _{\|x\|_{X} \leq 1}\|L(x)\|_{Y}$. An operator $K: X \rightarrow Y$ is called compact if it maps bounded subsets of $X$ into relatively compact subsets of $Y$.

Essential norm of a bounded operator $L: X \rightarrow Y$ is defined by

$$
\|L\|_{e, X \rightarrow Y}:=\inf _{K \in \mathcal{K}(X, Y)}\|L-K\|_{X \rightarrow Y}=\inf _{K \in \mathcal{K}(X, Y)} \sup _{\|x\|_{X} \leq 1}\|L(x)-K(x)\|_{Y}
$$

that is, as the distance of operator $L$ to the set of compact operators $\mathcal{K}(X, Y)$.

Let

$$
(D f)(z)=f^{\prime}(z)
$$

be the standard differentiation operator on $H(\mathbb{D})$. By $D^{k}$ we will denote the composition of (exactly) $k$ differentiation operators, that is, if $f \in H(\mathbb{D})$, then

$$
D^{k} f=\underbrace{D(D(\cdots(D f) \cdots))}_{k \text {-times }} \text {. }
$$

Let

$$
I_{k}(f)(z):=\int_{0}^{z} \int_{0}^{\zeta_{k}} \cdots \int_{0}^{\zeta_{2}} f\left(\zeta_{1}\right) d \zeta_{1} d \zeta_{2} \cdots d \zeta_{k}
$$

where $k \in \mathbb{N}$ and $f \in H(\mathbb{D})$.

It is clear that $D^{k} I_{k} f=f$ for every $f \in H(\mathbb{D})$, that is,

$$
D^{k} I_{k}=I d_{H(\mathbb{D})},
$$

where $I d_{X}$ denotes the identity operator on space $X$.

It is also easy to see that

$$
D^{j} I_{k}(f)(0)=0, \quad \text { for } j=\overline{0, k-1},
$$

where we regard that $D^{0}$ is the identity operator.

Beside this, by using the Newton-Leibnitz-type formula for holomorphic functions $k$ times, we have

$$
\begin{aligned}
I_{k} D^{k}(f)(z) & =\int_{0}^{z} \int_{0}^{\zeta_{k}} \cdots \int_{0}^{\zeta_{2}} f^{(k)}\left(\zeta_{1}\right) d \zeta_{1} d \zeta_{2} \cdots d \zeta_{k} \\
& =f(z)-\sum_{j=0}^{k-1} \frac{f^{(j)}(0)}{j !} z^{j},
\end{aligned}
$$

where $k \in \mathbb{N}$ and $f \in H(\mathbb{D})$, from which it follows that

$$
I_{k} D^{k} f=f
$$

for every $f \in H(\mathbb{D}) / \mathbb{P}_{k-1}$, that is, $I_{k} D^{k}$ is the identity operator on $H(\mathbb{D}) / \mathbb{P}_{k-1}$, and consequently on its subspaces, such as are $\mathcal{W}_{\mu}^{(m)} / \mathbb{P}_{k-1}$, where $m \geq k$. 
Let $\varphi \in S(\mathbb{D})$. Then by $C_{\varphi}$ we denote the composition operator on $H(\mathbb{D})$, which is defined by $C_{\varphi}(f)(z)=f(\varphi(z))$.

Let $u \in H(\mathbb{D})$. Then by $M_{g}$ is denoted the multiplication operator on $H(\mathbb{D})$, which is defined by $M_{g}(f)(z)=g(z) f(z)$.

The product of operators $C_{\varphi}$ and $M_{g}$, that is,

$$
\left(M_{g} \circ C_{\varphi}\right)(f)(z)=g(z) f(\varphi(z))
$$

is called the weighted composition operator and is denoted by $g C_{\varphi}$.

These three operators have been considerably studied on various spaces of holomorphic functions (see, for example, $[1,2,6,11,12]$ and the references therein).

Let $\varphi \in S(\mathbb{D}), g \in H(\mathbb{D})$ and $k \in \mathbb{N}$. We define an operator on $H(\mathbb{D})$ as follows:

$$
C_{\varphi, k}^{g}(f)(z):=\int_{0}^{z} \int_{0}^{\zeta_{k}} \cdots \int_{0}^{\zeta_{2}} f^{(k)}\left(\varphi\left(\zeta_{1}\right)\right) g\left(\zeta_{1}\right) d \zeta_{1} d \zeta_{2} \cdots d \zeta_{k}
$$

for $f \in H(\mathbb{D})$. For $k=1$ is obtained the generalized composition operator in [9], which was later studied or generalized, for example, in [10,13-17]. For some related operators; see, also [18-28] and the references therein.

Note that from (9) it immediately follows that

$$
D^{j} C_{\varphi, k}^{g}(f)(0)=0, \quad \text { for } j=\overline{0, k-1}
$$

Motivated by $[9,29,30]$ here we calculate the essential norm of operator (9) between two $k$ th weighted-type spaces. For some related results see also [6, 31].

\section{Main results}

In this section we prove the main results in this paper.

Theorem 1 Assume that $\mu$ and $v$ are weights, $k, m \in \mathbb{N}_{0}$, and that the operator $L: \mathcal{W}_{\mu}^{(k)} \rightarrow$ $\mathcal{W}_{\nu}^{(m)}$ is bounded. Then

$$
\|L\|_{e, \mathcal{W}_{\mu}^{(k)} \rightarrow \mathcal{W}_{v}^{(m)}}=\|L\|_{e, \mathcal{W}_{\mu}^{(k)} / \mathbb{P}_{k-1} \rightarrow \mathcal{W}_{v}^{(m)}}
$$

Proof If $k=0$, then we regard that $\mathcal{W}_{\mu}^{(0)} / \mathbb{P}_{-1}=\mathcal{W}_{\mu}^{(0)}$, so that (11) obviously holds. Now assume that $k \in \mathbb{N}$. For each compact operator $K: \mathcal{W}_{\mu}^{(k)} \rightarrow \mathcal{W}_{\nu}^{(m)}$, its restriction on $\mathcal{W}_{\mu}^{(k)} / \mathbb{P}_{k-1}$, that is, $K: \mathcal{W}_{\mu}^{(k)} / \mathbb{P}_{k-1} \rightarrow \mathcal{W}_{v}^{(m)}$, is also a compact operator, from which along with the definition of the essential norm of an operator, it easily follows that

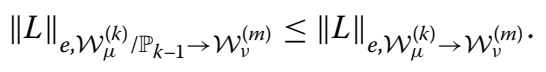

Let $K: \mathcal{W}_{\mu}^{(k)} / \mathbb{P}_{k-1} \rightarrow \mathcal{W}_{v}^{(m)}$ be a compact operator and $f \in \mathcal{W}_{\mu}^{(k)}$. Then by

$$
\widetilde{K}(f)(z)=K\left(f-\sum_{j=0}^{k-1} a_{j} z^{j}\right),
$$


where $a_{j}=f^{(j)}(0) / j !, j=\overline{0, k-1}$, is defined an extension of operator $K$ on the whole space $\mathcal{W}_{\mu}^{(k)}$, that is, $\widetilde{K}: \mathcal{W}_{\mu}^{(k)} \rightarrow \mathcal{W}_{v}^{(m)}$, which is obviously a compact operator. Denote the set of such obtained operators $\widetilde{K}$ by $\widetilde{\mathcal{K}}$.

Let $L_{1}: \mathcal{W}_{\mu}^{(k)} \rightarrow \mathcal{W}_{\mu}^{(m)}$ be a bounded operator, then the operator

$$
\widetilde{L}_{1}(f):=L_{1}\left(\sum_{j=0}^{k-1} a_{j} z^{j}\right)=\sum_{j=0}^{k-1} a_{j} L_{1}\left(z^{j}\right)
$$

where, as above, $a_{j}=f^{(j)}(0) / j !, j=\overline{0, k-1}$, maps $\mathcal{W}_{\mu}^{(k)}$ into $\mathcal{W}_{\mu}^{(m)}$, and is compact, since its image is a finite-dimensional space.

We have

$$
\begin{aligned}
& \|L\|_{e, \mathcal{W}_{\mu}^{(k)} \rightarrow \mathcal{W}_{v}^{(m)}} \\
& =\inf _{K_{1} \in \mathcal{K}\left(\mathcal{W}_{\mu}^{(k)}, \mathcal{W}_{v}^{(m)}\right)}\left\|L-K_{1}\right\|_{\mathcal{W}_{\mu}^{(k)} \rightarrow \mathcal{W}_{v}^{(m)}} \\
& \leq \inf _{\widetilde{K} \in \mathcal{K}\left(\mathcal{W}_{\mu}^{(k)}, \mathcal{W}_{v}^{(m)}\right) \cap \widetilde{\mathcal{K}}}\|L-\widetilde{K}-\widetilde{L}\|_{\mathcal{W}_{\mu}^{(k)} \rightarrow \mathcal{W}_{v}^{(m)}} \\
& =\inf _{\widetilde{K} \in \mathcal{K}\left(\mathcal{W}_{\mu}^{(k)}, \mathcal{W}_{v}^{(m)} \cap \cap \widetilde{\mathcal{K}}\|f\|_{\mathcal{W}_{\mu}^{(k)} \leq 1}\right.}\|L(f)-\widetilde{K}(f)-\widetilde{L}(f)\|_{\mathcal{W}_{v}^{(m)}} \\
& =\inf _{K \in \mathcal{K}\left(\mathcal{W}_{\mu}^{(k) / \mathbb{P}}{ }_{k-1}, \mathcal{W}_{v}^{(m)}\right)\|f\|_{\mathcal{W}_{\mu}^{(k)}} \leq 1}\left\|L(f)-K\left(f-\sum_{j=0}^{k-1} a_{j} z^{j}\right)-L\left(\sum_{j=0}^{k-1} a_{j} z^{j}\right)\right\|_{\mathcal{W}_{v}^{(m)}} \\
& =\inf _{K \in \mathcal{K}\left(\mathcal{W}_{\mu}^{(k)} / \mathbb{P}_{k-1}, \mathcal{W}_{v}^{(m)}\right)\|f\|_{\mathcal{W}_{\mu}^{(k)}}} \sup _{\|}\left\|(L-K)\left(f-\sum_{j=0}^{k-1} a_{j} z^{j}\right)\right\|_{\mathcal{W}_{v}^{(m)}} \\
& \leq \inf _{K \in \mathcal{K}\left(\mathcal{W}_{\mu}^{(k)} / \mathbb{P}_{k-1}, \mathcal{W}_{v}^{(m)}\right)} \sup _{\left\{g \in \mathcal{W}_{\mu}^{(k)} \mathbb{P}_{k-1}:\|g\| \|_{\mathcal{W}_{\mu}^{(k)}} \leq 1\right\}}\|(L-K)(g)\|_{\mathcal{W}_{\nu}^{(m)}}
\end{aligned}
$$

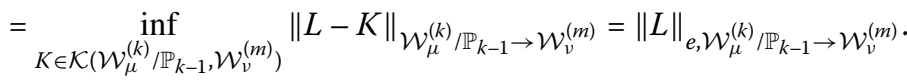

From (12) and (13), equality (11) follows.

Theorem 2 Assume that $\mu$ and $v$ are weights, $k, l, m \in \mathbb{N}, m \geq k$, and that the operator $C_{\varphi, k}^{g}: \mathcal{W}_{\mu}^{(m+l-1)} \rightarrow \mathcal{W}_{v}^{(m)}$ is bounded. Then

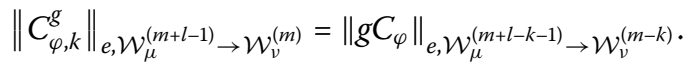

Proof First we prove the following inequality:

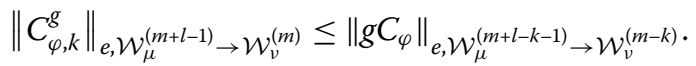

We show that

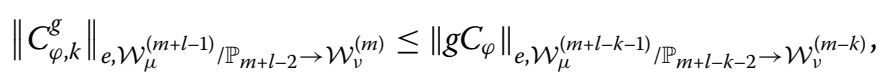


which by Theorem 1 is equivalent to (15) (recall that when $m=k$ and $l=1$, we naturally regard that $\left.\mathcal{W}_{\mu}^{(m+l-k-1)} / \mathbb{P}_{m+l-k-2}=\mathcal{W}_{\mu}^{(0)}\right)$.

Assume that $f \in \mathcal{W}_{\mu}^{(m+l-k-1)} / \mathbb{P}_{m+l-k-2}$. Then, since

$$
\left\|I_{k} f\right\|_{\mathcal{W}_{\mu}^{(m+l-1)}}=\|f\|_{\mathcal{W}_{\mu}^{(m+l-k-1)}<+\infty}
$$

and

$$
D^{j}\left(I_{k} f\right)(0)=0, \quad \text { for } j=\overline{0, m+l-2} \text {, }
$$

it follows that $I_{k} f \in \mathcal{W}_{\mu}^{(m+l-1)} / \mathbb{P}_{m+l-2}$, that is, operator $I_{k}$ maps the space $\mathcal{W}_{\mu}^{(m+l-k-1)} / \mathbb{P}_{m+l-k-2}$ into $\mathcal{W}_{\mu}^{(m+l-1)} / \mathbb{P}_{m+l-2}$. Further, it is clear that $C_{\varphi, k}^{g}: \mathcal{W}_{\mu}^{(m+l-1)} / \mathbb{P}_{m+l-2} \rightarrow \mathcal{W}_{\nu}^{(m)}$ is bounded, and since for every $h \in \mathcal{W}_{v}^{(m)}$,

$$
b_{\mathcal{W}_{v}^{(m-k)}}\left(D^{k} h\right)=b_{\mathcal{W}_{v}^{(m)}}(h)<\infty
$$

we see that $D^{k}$ maps $\mathcal{W}_{v}^{(m)}$ into $\mathcal{W}_{v}^{(m-k)}$. Moreover, we have

$$
\left\|D^{k} h\right\|_{\mathcal{W}_{v}^{(m-k)}} \leq\|h\|_{\mathcal{W}_{v}^{(m)}},
$$

where the strict inequality can occur here.

Hence, we see that the operator

$$
D^{k} C_{\varphi, k}^{g} I_{k}=g C_{\varphi}
$$

maps the space $\mathcal{W}_{\mu}^{(m+l-k-1)} / \mathbb{P}_{m+l-k-2}$ into $\mathcal{W}_{v}^{(m-k)}$, and from (17), (20) and the boundedness of the operator $C_{\varphi, k}^{g}: \mathcal{W}_{\mu}^{(m+l-1)} / \mathbb{P}_{m+l-2} \rightarrow \mathcal{W}_{\nu}^{(m)}$, it follows that the operator $g C_{\varphi}$ : $\mathcal{W}_{\mu}^{(m+l-k-1)} / \mathbb{P}_{m+l-k-2} \rightarrow \mathcal{W}_{v}^{(m-k)}$ is also bounded.

Due to (8) and (10), we have

$$
I_{k} D^{k} C_{\varphi, k}^{g} I_{k} D^{k} f=C_{\varphi, k}^{g} f
$$

for every $f \in \mathcal{W}_{\mu}^{(m+l-1)} / \mathbb{P}_{m+l-2}$. Indeed, since $m \geq k$ and $l \in \mathbb{N}$ we have $m+l-1 \geq k$, so by (8) we have $I_{k} D^{k} f=f$, and further from (10) we get $C_{\varphi, k}^{g} f \in \mathcal{W}_{\mu}^{(m)} / \mathbb{P}_{k-1}$. By another application of (8) is obtained (22).

Let $K: \mathcal{W}_{\mu}^{(m+l-k-1)} / \mathbb{P}_{m+l-k-2} \rightarrow \mathcal{W}_{\nu}^{(m-k)}$ be a compact operator and

$$
\widetilde{K}:=I_{k} K D^{k} \text {. }
$$

Then the operator maps the space $\mathcal{W}_{\mu}^{(m+l-1)} / \mathbb{P}_{m+l-2}$ into $\mathcal{W}_{\nu}^{(m)}$ and is compact, since the space of compact operators is a both sided ideal into the space of bounded linear operators. 
Hence, by using (21)-(23) and some simple estimates, we have

$$
\begin{aligned}
& \left\|C_{\varphi, k}^{g} f-\widetilde{K} f\right\|_{\mathcal{W}_{v}^{(m)}}=\left\|I_{k} D^{k} C_{\varphi, k}^{g} I_{k} D^{k} f-I_{k} K D^{k} f\right\|_{\mathcal{W}_{v}^{(m)}} \\
& =\left\|I_{k}\left(D^{k} C_{\varphi, k}^{g} I_{k}-K\right) D^{k} f\right\|_{\mathcal{W}_{v}^{(m)}} \\
& =\left\|\left(g C_{\varphi}-K\right) D^{k} f\right\|_{\mathcal{W}_{\nu}^{(m-k)}}
\end{aligned}
$$

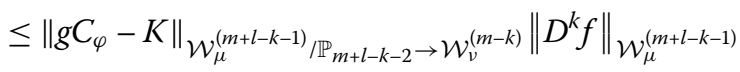

$$
\begin{aligned}
& \leq\left\|g C_{\varphi}-K\right\|_{\mathcal{W}_{\mu}^{(m+l-k-1)} / \mathbb{P}_{m+l-k-2} \rightarrow \mathcal{W}_{\nu}^{(m-k)}}\|f\|_{\mathcal{W}_{\mu}^{(m+l-1)}} .
\end{aligned}
$$

By taking the supremum in (24) over the unit ball in $\mathcal{W}_{\mu}^{(m+l-1)} / \mathbb{P}_{m+l-2}$, and then taking the infimum in such obtained inequality over the set of all compact operators $K$ : $\mathcal{W}_{\mu}^{(m+l-k-1)} / \mathbb{P}_{m+l-k-2} \rightarrow \mathcal{W}_{v}^{(m-k)}$, we get (16).

Now we prove the following inequality:

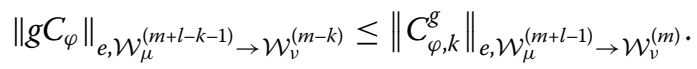

To do this first note that since (17) holds for every $f \in \mathcal{W}_{\mu}^{(m+l-k-1)}$, the operator $I_{k}$ : $\mathcal{W}_{\mu}^{(m+l-k-1)} \rightarrow \mathcal{W}_{\mu}^{(m+l-1)}$ is bounded. From this, since $C_{\varphi, k}^{g}: \mathcal{W}_{\mu}^{(m+l-1)} \rightarrow \mathcal{W}_{\nu}^{(m)}$ is bounded by the assumption, and $D^{k}: \mathcal{W}_{\mu}^{(m)} \rightarrow \mathcal{W}_{\nu}^{(m-k)}$ is also bounded due to the inequality in (20), we see that the operator $D^{k} C_{\varphi, k}^{g} I_{k}=g C_{\varphi}: \mathcal{W}_{\mu}^{(m+l-k-1)} \rightarrow \mathcal{W}_{v}^{(m-k)}$ is bounded.

Note also that

$$
C_{\varphi, k}^{g}=I_{k} g C_{\varphi} D^{k}
$$

where $D^{k}: \mathcal{W}_{\mu}^{(m+l-1)} \rightarrow \mathcal{W}_{\mu}^{(m+l-k-1)}$ and $I_{k}: \mathcal{W}_{\nu}^{(m-k)} \rightarrow \mathcal{W}_{\nu}^{(m)}$.

Let $K: \mathcal{W}_{\mu}^{(m+l-1)} \rightarrow \mathcal{W}_{v}^{(m)}$ be a compact operator. Then the operator

$$
D^{k} K I_{k}: \mathcal{W}_{\mu}^{(m+l-k-1)} \rightarrow \mathcal{W}_{v}^{(m-k)}
$$

is compact too.

Using this facts and (5), it follows that

$$
\begin{aligned}
\left\|g C_{\varphi} f-D^{k} K I_{k} f\right\|_{\mathcal{W}_{v}^{(m-k)}} & =\left\|D^{k} I_{k} g C_{\varphi} D^{k} I_{k} f-D^{k} K I_{k} f\right\|_{\mathcal{W}_{v}^{(m-k)}} \\
& =\left\|D^{k}\left(C_{\varphi, k}^{g}-K\right) I_{k} f\right\|_{\mathcal{W}_{v}^{(m-k)}} \\
& \leq\left\|\left(C_{\varphi, k}^{g}-K\right) I_{k} f\right\|_{\mathcal{W}_{v}^{(m)}} \\
& \leq\left\|C_{\varphi, k}^{g}-K\right\|_{\mathcal{W}_{\mu}^{(m+l-1)} \rightarrow \mathcal{W}_{v}^{(m)}}\left\|I_{k} f\right\|_{\mathcal{W}_{\mu}^{(m+l-1)}} \\
& \leq\left\|C_{\varphi, k}^{g}-K\right\|_{\mathcal{W}_{\mu}^{(m+l-1)} \rightarrow \mathcal{W}_{v}^{(m)}}\|f\|_{\mathcal{W}_{\mu}^{(m+l-k-1)}} .
\end{aligned}
$$

By taking the supremum in (27) over the unit ball in $\mathcal{W}_{\mu}^{(m+l-k-1)}$, and then taking the infimum in such obtained inequality over the set of all compact operators $K: \mathcal{W}_{\mu}^{(m+l-1)} \rightarrow$ $\mathcal{W}_{\nu}^{(m)}$, the inequality (25) is obtained. From (15) and (25) equality (14) follows. 
Before we formulate our next results, we want to say that their proofs are related to the one of Theorem 2, but we will give all the differences for the completeness.

Theorem 3 Assume that $\mu$ and $v$ are weights, $k, l, m \in \mathbb{N}, m \geq k$, and that the operator $C_{\varphi, k}^{g}: \mathcal{W}_{\mu}^{(m)} \rightarrow \mathcal{W}_{\nu}^{(m+l-1)}$ is bounded. Then

$$
\left\|C_{\varphi, k}^{g}\right\|_{e, \mathcal{W}_{\mu}^{(m)} \rightarrow \mathcal{W}_{v}^{(m+l-1)}}=\left\|g C_{\varphi}\right\|_{e, \mathcal{W}_{\mu}^{(m-k)} \rightarrow \mathcal{W}_{v}^{(m+l-k-1)}} .
$$

Proof First we prove

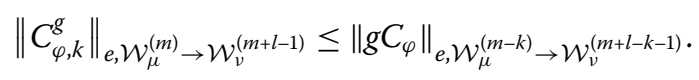

We show that

$$
\left\|C_{\varphi, k}^{g}\right\|_{e, \mathcal{W}_{\mu}^{(m)}} \mathbb{P}_{m-1} \rightarrow \mathcal{W}_{v}^{(m+l-1)} \leq\left\|g C_{\varphi}\right\|_{e, \mathcal{W}_{\mu}^{(m-k)} / \mathbb{P}_{m-k-1} \rightarrow \mathcal{W}_{v}^{(m+l-k-1)},}
$$

which by Theorem 1 is equivalent to (29).

Assume that $f \in \mathcal{W}_{\mu}^{(m-k)} / \mathbb{P}_{m-k-1}$. Then, since

$$
\left\|I_{k} f\right\|_{\mathcal{W}_{\mu}^{(m)}}=\|f\|_{\mathcal{W}_{\mu}^{(m-k)}}<\infty
$$

and

$$
D^{j}\left(I_{k} f\right)(0)=0, \quad \text { for } j=\overline{0, m-1},
$$

it follows that $I_{k} f \in \mathcal{W}_{\mu}^{(m)} / \mathbb{P}_{m-1}$, that is, operator $I_{k}$ maps space $\mathcal{W}_{\mu}^{(m-k)} / \mathbb{P}_{m-k-1}$ into $\mathcal{W}_{\mu}^{(m)} / \mathbb{P}_{m-1}$. Further, it is clear that $C_{\varphi, k}^{g}: \mathcal{W}_{\mu}^{(m)} / \mathbb{P}_{m-1} \rightarrow \mathcal{W}_{\nu}^{(m+l-1)}$ is bounded, and since for every $h \in \mathcal{W}_{v}^{(m+l-1)}$,

$$
b_{\mathcal{W}_{\mu}^{(m+l-k-1)}}\left(D^{k} h\right)=b_{\mathcal{W}_{\mu}^{(m+l-1)}}(h)<+\infty
$$

we see that $D^{k}: \mathcal{W}_{v}^{(m+l-1)} \rightarrow \mathcal{W}_{v}^{(m+l-k-1)}$. Moreover, we have

$$
\left\|D^{k} h\right\|_{\mathcal{W}_{\mu}^{(m+l-k-1)}} \leq\|h\|_{\mathcal{W}_{\mu}^{(m+l-1)}}
$$

Hence, we see that the operator (21) maps $\mathcal{W}_{\mu}^{(m-k)} / \mathbb{P}_{m-k-1}$ to $\mathcal{W}^{(m+l-k-1)}$, and from (31), (34) and the boundedness of $C_{\varphi, k}^{g}: \mathcal{W}_{\mu}^{(m)} / \mathbb{P}_{m-1} \rightarrow \mathcal{W}_{\nu}^{(m+l-1)}$ it follows that the operator $g C_{\varphi}$ : $\mathcal{W}_{\mu}^{(m-k)} / \mathbb{P}_{m-k-1} \rightarrow \mathcal{W}^{(m+l-k-1)}$ is also bounded. Beside this, since $m \geq k$, we see that (22) holds for every $f \in \mathcal{W}_{\mu}^{(m)} / \mathbb{P}_{m-1}$.

Let $K: \mathcal{W}_{\mu}^{(m-k)} / \mathbb{P}_{m-k-1} \rightarrow \mathcal{W}_{v}^{(m+l-k-1)}$ be a compact operator. Then the operator

$$
\widetilde{K}:=I_{k} K D^{k}: \mathcal{W}_{\mu}^{(m)} / \mathbb{P}_{m-1} \rightarrow \mathcal{W}_{v}^{(m+l-1)}
$$

is compact. 
From this, (21) and (22), we have

$$
\begin{aligned}
\left\|C_{\varphi, k}^{g} f-\widetilde{K} f\right\|_{\mathcal{W}_{v}^{(m+l-1)}} & =\left\|I_{k} D^{k} C_{\varphi, k}^{g} I_{k} D^{k} f-I_{k} K D^{k} f\right\|_{\mathcal{W}_{v}^{(m+l-1)}} \\
& =\left\|I_{k}\left(D^{k} C_{\varphi, k}^{g} I_{k}-K\right) D^{k} f\right\|_{\mathcal{W}_{\nu}^{(m+l-1)}} \\
& =\left\|\left(g C_{\varphi}-K\right) D^{k} f\right\|_{\mathcal{W}_{v}^{(m+l-k-1)}} \\
& \leq\left\|g C_{\varphi}-K\right\|_{\mathcal{W}_{\mu}^{(m-k)} / \mathbb{P}_{m-k-1} \rightarrow \mathcal{W}^{(m+l-k-1)}}\left\|D^{k} f\right\|_{\mathcal{W}_{\mu}^{(m-k)} / \mathbb{P}_{m-k-1}} \\
& \leq\left\|g C_{\varphi}-K\right\|_{\mathcal{W}_{\mu}^{(m-k)} / \mathbb{P}_{m-k-1} \rightarrow \mathcal{W}^{(m+l-k-1)}}\|f\|_{\mathcal{W}_{\mu}^{(m)} .}
\end{aligned}
$$

By taking the supremum in (35) over the unit ball in $\mathcal{W}_{\mu}^{(m)} / \mathbb{P}_{m-1}$, and then taking the infimum in such obtained inequality over the set of all compact operators $K$ : $\mathcal{W}_{\mu}^{(m-k)} / \mathbb{P}_{m-k-1} \rightarrow \mathcal{W}_{v}^{(m+l-k-1)}$, we get (30).

Now we prove that

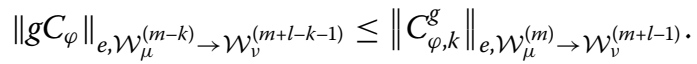

Since (31) holds for every $f \in \mathcal{W}_{\mu}^{(m-k)}$, we see that the operator $I_{k}: \mathcal{W}_{\mu}^{(m-k)} \rightarrow \mathcal{W}_{\mu}^{(m)}$ is bounded. From this, since $C_{\varphi, k}^{g}: \mathcal{W}_{\mu}^{(m)} \rightarrow \mathcal{W}_{\nu}^{(m+l-1)}$ is bounded by the assumption, and $D^{k}: \mathcal{W}_{v}^{(m+l-1)} \rightarrow \mathcal{W}_{v}^{(m+l-k-1)}$ is also bounded due to the inequality in (34), we see that the operator $D^{k} C_{\varphi, k}^{g} I_{k}=g C_{\varphi}: \mathcal{W}_{\mu}^{(m-k)} \rightarrow \mathcal{W}_{\nu}^{(m+l-k-1)}$ is bounded. Note also that (26) holds, where $D^{k}: \mathcal{W}_{\mu}^{(m)} \rightarrow \mathcal{W}_{v}^{(m-k)}$ and $I_{k}: \mathcal{W}_{\mu}^{(m+l-k-1)} \rightarrow \mathcal{W}_{v}^{(m+l-1)}$.

Let $K: \mathcal{W}_{\mu}^{(m)} \rightarrow \mathcal{W}_{v}^{(m+l-1)}$ be a compact operator. Then the operator

$$
D^{k} K I_{k}: \mathcal{W}_{\mu}^{(m-k)} \rightarrow \mathcal{W}_{v}^{(m+l-k-1)}
$$

is compact.

Using this fact along with (5) and (26), we have

$$
\begin{aligned}
\left\|g C_{\varphi} f-D^{k} K I_{k} f\right\|_{\mathcal{W}_{v}^{(m+l-k-1)}} & =\left\|D^{k} I_{k} g C_{\varphi} D^{k} I_{k} f-D^{k} K I_{k} f\right\|_{\mathcal{W}_{v}^{(m+l-k-1)}} \\
& =\left\|D^{k}\left(C_{\varphi, k}^{g}-K\right) I_{k} f\right\|_{\mathcal{W}_{v}^{(m+l-k-1)}} \\
& \leq\left\|\left(C_{\varphi, k}^{g}-K\right) I_{k} f\right\|_{\mathcal{W}_{v}^{(m+l-1)}} \\
& \leq\left\|C_{\varphi, k}^{g}-K\right\|_{\mathcal{W}_{\mu}^{(m)} \rightarrow \mathcal{W}_{v}^{(m+l-1)}}\left\|I_{k} f\right\|_{\mathcal{W}_{\mu}^{(m)}} \\
& \leq\left\|C_{\varphi, k}^{g}-K\right\|_{\mathcal{W}_{\mu}^{(m)} \rightarrow \mathcal{W}_{v}^{(m+l-1)}}\|f\|_{\mathcal{W}_{\mu}^{(m-k)}} .
\end{aligned}
$$

By taking the supremum in (37) over the unit ball in $\mathcal{W}_{\mu}^{(m-k)}$, and then taking the infimum in such obtained inequality over the set of all compact operators $K: \mathcal{W}_{\mu}^{(m)} \rightarrow \mathcal{W}_{\nu}^{(m+l-1)}$, we get (36). From (29) and (36) is directly obtained (28), as desired.

Theorem 4 Assume that $\mu$ and $v$ are weights, $k, l, m \in \mathbb{N}, m \geq k$, and that the operator $C_{\varphi, k}^{g}: \mathcal{W}_{\mu}^{(m+l-1)} / \mathbb{P}_{m+l-2} \rightarrow \mathcal{W}_{\nu}^{(m)} / \mathbb{P}_{m-1}$ is bounded. Then

$$
\left\|C_{\varphi, k}^{g}\right\|_{e, \mathcal{W}_{\mu}^{(m+l-1)} / \mathbb{P}_{m+l-2} \rightarrow \mathcal{W}_{\nu}^{(m)} / \mathbb{P}_{m-1}}=\left\|g C_{\varphi}\right\|_{e, \mathcal{W}_{\mu}^{(m+l-k-1)} / \mathbb{P}_{m+l-k-2} \rightarrow \mathcal{W}_{\nu}^{(m-k)} / \mathbb{P}_{m-k-1}} .
$$


Proof First we prove the following inequality:

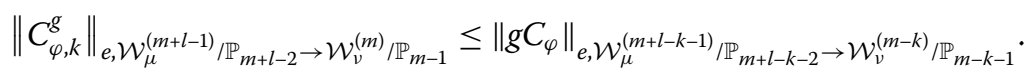

Assume that $f \in \mathcal{W}_{\mu}^{(m+l-k-1)} / \mathbb{P}_{m+l-k-2}$. Then, since (17) and (18) hold, we see that the operator $I_{k}$ maps the space $\mathcal{W}_{\mu}^{(m+l-k-1)} / \mathbb{P}_{m+l-k-2}$ into $\mathcal{W}_{\mu}^{(m+l-1)} / \mathbb{P}_{m+l-2}$. Further, by the assumption $C_{\varphi, k}^{g}: \mathcal{W}_{\mu}^{(m+l-1)} / \mathbb{P}_{m+l-2} \rightarrow \mathcal{W}_{v}^{(m)} / \mathbb{P}_{m-1}$ is bounded, and since for every $h \in \mathcal{W}_{v}^{(m)} / \mathbb{P}_{m-1}$, (19) holds and

$$
D^{j}\left(D^{k} h\right)(0)=0, \quad j=\overline{0, m-k-1},
$$

we have $D^{k}: \mathcal{W}_{v}^{(m)} / \mathbb{P}_{m-1} \rightarrow \mathcal{W}_{v}^{(m-k)} / \mathbb{P}_{m-k-1}$. Moreover, we see that (20) holds.

Hence, we see that the operator (21) maps the space $\mathcal{W}_{\mu}^{(m+l-k-1)} / \mathbb{P}_{m+l-k-2}$ into $\mathcal{W}_{v}^{(m-k)} / \mathbb{P}_{m-k-1}$, and from (17), (20) and the boundedness of the operator $C_{\varphi, k}^{g}$ : $\mathcal{W}_{\mu}^{(m+l-1)} / \mathbb{P}_{m+l-2} \rightarrow \mathcal{W}_{v}^{(m)} / \mathbb{P}_{m-1}$, it follows that $g C_{\varphi}: \mathcal{W}_{\mu}^{(m+l-k-1)} / \mathbb{P}_{m+l-k-2} \rightarrow \mathcal{W}_{v}^{(m-k)} / \mathbb{P}_{m-k-1}$ is also bounded. Since $m+l-1 \geq k$, we see that (22) holds for every $f \in \mathcal{W}_{\mu}^{(m+l-1)} / \mathbb{P}_{m+l-2}$.

Let $K: \mathcal{W}_{\mu}^{(m+l-k-1)} / \mathbb{P}_{m+l-k-2} \rightarrow \mathcal{W}_{v}^{(m-k)} / \mathbb{P}_{m-k-1}$ be a compact operator and $\widetilde{K}$ be defined as in (23), where $D^{k}$ maps the space $\mathcal{W}_{\mu}^{(m+l-1)} / \mathbb{P}_{m+l-2}$ into $\mathcal{W}_{\mu}^{(m+l-k-1)} / \mathbb{P}_{m+l-k-2}$, and $I_{k}$ maps the space $\mathcal{W}_{v}^{(m-k)} / \mathbb{P}_{m-k-1}$ into $\mathcal{W}_{v}^{(m)} / \mathbb{P}_{m-1}$. Then, $\widetilde{K}$ maps the space $\mathcal{W}_{\mu}^{(m+l-1)} / \mathbb{P}_{m+l-2}$ into $\mathcal{W}_{v}^{(m)} / \mathbb{P}_{m-1}$ and is compact.

From this and (21), similar to (24), is obtained

$$
\left\|C_{\varphi, k}^{g} f-\widetilde{K} f\right\|_{\mathcal{W}_{v}^{(m)}} \leq\left\|g C_{\varphi}-K\right\|_{\mathcal{W}_{\mu}^{(m+l-k-1)} \mathbb{P}_{m+l-k-2} \rightarrow \mathcal{W}_{v}^{(m-k)} \mathbb{P}_{m-k-1}}\|f\|_{\mathcal{W}_{\mu}^{(m+l-1)}} .
$$

By taking the supremum in (41) over the unit ball in $\mathcal{W}_{\mu}^{(m+l-1)} / \mathbb{P}_{m+l-2}$, and then taking the infimum in such obtained inequality over the set of all compact operators $K$ : $\mathcal{W}_{\mu}^{(m+l-k-1)} / \mathbb{P}_{m+l-k-2} \rightarrow \mathcal{W}_{v}^{(m-k)} / \mathbb{P}_{m-k-1}$, we get (39).

Now we prove that

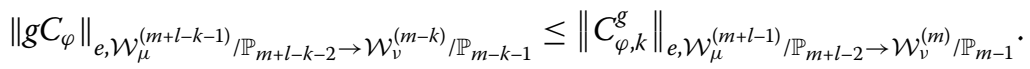

Since (17) holds for every $f \in \mathcal{W}_{\mu}^{(m+l-k-1)} / \mathbb{P}_{m+l-k-2}$, the operator $I_{k}: \mathcal{W}_{\mu}^{(m+l-k-1) /}$ $\mathbb{P}_{m+l-k-2} \rightarrow \mathcal{W}_{\mu}^{(m+l-1)} / \mathbb{P}_{m+l-2}$ is bounded. From this, since $C_{\varphi, k}^{g}: \mathcal{W}_{\mu}^{(m+l-1)} / \mathbb{P}_{m+l-2} \rightarrow$ $\mathcal{W}_{v}^{(m)} / \mathbb{P}_{m-1}$ is bounded, and since $D^{k}: \mathcal{W}_{\mu}^{(m)} / \mathbb{P}_{m-1} \rightarrow \mathcal{W}_{v}^{(m-k)} / \mathbb{P}_{m-k-1}$ is also bounded due to (20) and (40), we see that the operator $D^{k} C_{\varphi, k}^{g} I_{k}=g C_{\varphi}: \mathcal{W}_{\mu}^{(m+l-k-1)} / \mathbb{P}_{m+l-k-2} \rightarrow$ $\mathcal{W}_{v}^{(m-k)} / \mathbb{P}_{m-k-1}$ is bounded. Beside this, note that $C_{\varphi, k}^{g}=I_{k} g C_{\varphi} D^{k}: \mathcal{W}_{\mu}^{(m+l-1)} / \mathbb{P}_{m+l-2} \rightarrow$ $\mathcal{W}_{v}^{(m)} / \mathbb{P}_{m-1}$, where $D^{k}: \mathcal{W}_{\mu}^{(m+l-1)} / \mathbb{P}_{m+l-2} \rightarrow \mathcal{W}_{\mu}^{(m+l-k-1)} / \mathbb{P}_{m+l-k-2}$ and $I_{k}: \mathcal{W}_{v}^{(m-k)} / \mathbb{P}_{m-k-1} \rightarrow$ $\mathcal{W}_{v}^{(m)} / \mathbb{P}_{m-1}$.

Let $K: \mathcal{W}_{\mu}^{(m+l-1)} / \mathbb{P}_{m+l-2} \rightarrow \mathcal{W}_{v}^{(m)} / \mathbb{P}_{m-1}$ be a compact operator. Then the operator

$$
D^{k} K I_{k}: \mathcal{W}_{\mu}^{(m+l-k-1)} / \mathbb{P}_{m+l-k-2} \rightarrow \mathcal{W}_{v}^{(m-k)} / \mathbb{P}_{m-k-1}
$$

is compact too.

Hence, as in (27) we have

$$
\left\|g C_{\varphi} f-D^{k} K I_{k} f\right\|_{\mathcal{W}_{v}^{(m-k)}} \leq\left\|C_{\varphi, k}^{g}-K\right\|_{\mathcal{W}_{\mu}^{(m+l-1)} / \mathbb{P}_{m+l-2} \rightarrow \mathcal{W}_{v}^{(m)} / \mathbb{P}_{m-1}}\|f\|_{\mathcal{W}_{\mu}^{(m+l-k-1)}} .
$$


By taking the supremum in (43) over the unit ball in $\mathcal{W}_{\mu}^{(m+l-k-1)} / \mathbb{P}_{m+l-k-2}$, and then taking the infimum in such obtained inequality over the set of all compact operators $K: \mathcal{W}_{\mu}^{(m+l-1)} / \mathbb{P}_{m+l-2} \rightarrow \mathcal{W}_{\nu}^{(m)} / \mathbb{P}_{m-1}$, we get (42). From (39) and (42) is obtained (38).

Theorem 5 Assume that $\mu$ and $v$ are weights, $k, l, m \in \mathbb{N}, m \geq k$, and that the operator $C_{\varphi, k}^{g}: \mathcal{W}_{\mu}^{(m)} / \mathbb{P}_{m-1} \rightarrow \mathcal{W}_{\nu}^{(m+l-1)} / \mathbb{P}_{m+l-2}$ is bounded. Then

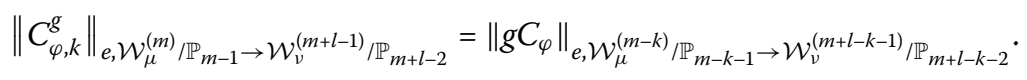

Proof First we prove that

$$
\left\|C_{\varphi, k}^{g}\right\|_{e, \mathcal{W}_{\mu}^{(m)} / \mathbb{P}_{m-1} \rightarrow \mathcal{W}_{v}^{(m+l-1)} / \mathbb{P}_{m+l-2}} \leq\left\|g C_{\varphi}\right\|_{e, \mathcal{W}_{\mu}^{(m-k)} / \mathbb{P}_{m-k-1} \rightarrow \mathcal{W}_{v}^{(m+l-k-1)} / \mathbb{P}_{m+l-k-2}}
$$

Assume that $f \in \mathcal{W}_{\mu}^{(m-k)} / \mathbb{P}_{m-k-1}$. Recall that, since (31) and (32) hold, the operator $I_{k}$ boundedly maps space $\mathcal{W}_{\mu}^{(m-k)} / \mathbb{P}_{m-k-1}$ into $\mathcal{W}_{\mu}^{(m)} / \mathbb{P}_{m-1}$. Since equality (33) holds for every $h \in \mathcal{W}_{v}^{(m+l-1)} / \mathbb{P}_{m+l-2}$ and

$$
D^{j}\left(D^{k} h\right)(0)=0, \quad \overline{0, m+l-k-2},
$$

we see that $D^{k}: \mathcal{W}_{v}^{(m+l-1)} / \mathbb{P}_{m+l-2} \rightarrow \mathcal{W}_{v}^{(m+l-k-1)} / \mathbb{P}_{m+l-k-2}$. Moreover, we see that (34) holds.

Using these two facts and the boundedness of the operator $C_{\varphi, k}^{g}: \mathcal{W}_{\mu}^{(m)} / \mathbb{P}_{m-1} \rightarrow$ $\mathcal{W}_{\nu}^{(m+l-1)} / \mathbb{P}_{m+l-2}$, we see that the operator (21) maps the space $\mathcal{W}_{\mu}^{(m-k)} / \mathbb{P}_{m-k-1}$ to $\mathcal{W}^{(m+l-k-1)} /$ $\mathbb{P}_{m+l-k-2}$, and from (31), (34) and the boundedness of the operator $C_{\varphi, k}^{g}: \mathcal{W}_{\mu}^{(m)} / \mathbb{P}_{m-1} \rightarrow$ $\mathcal{W}_{v}^{(m+l-1)} / \mathbb{P}_{m+l-2}$, it follows that the operator $g C_{\varphi}: \mathcal{W}_{\mu}^{(m-k)} / \mathbb{P}_{m-k-1} \rightarrow \mathcal{W}^{(m+l-k-1)} / \mathbb{P}_{m+l-k-2}$ is also bounded. We also see that (22) holds for every $f \in \mathcal{W}_{\mu}^{(m)} / \mathbb{P}_{m-1}$.

Let $K: \mathcal{W}_{\mu}^{(m-k)} / \mathbb{P}_{m-k-1} \rightarrow \mathcal{W}_{v}^{(m+l-k-1)} / \mathbb{P}_{m+l-k-2}$ be a compact operator. Then the operator

$$
\widetilde{K}:=I_{k} K D^{k}: \mathcal{W}_{\mu}^{(m)} / \mathbb{P}_{m-1} \rightarrow \mathcal{W}_{\nu}^{(m+l-1)} / \mathbb{P}_{m+l-2}
$$

is compact.

Hence, as in (35) we have

$$
\left\|C_{\varphi, k}^{g} f-\widetilde{K} f\right\|_{\mathcal{W}_{v}^{(m+l-1)}} \leq\left\|g C_{\varphi}-K\right\|_{\mathcal{W}_{\mu}^{(m-k)} / \mathbb{P}_{m-k-1} \rightarrow \mathcal{W}^{(m+l-k-1)} / \mathbb{P}_{m+l-k-2}}\|f\|_{\mathcal{W}_{\mu}^{(m)}} .
$$

By taking the supremum in (47) over the unit ball in $\mathcal{W}_{\mu}^{(m)} / \mathbb{P}_{m-1}$, and then taking the infimum in such obtained inequality over the set of all compact operators $K$ : $\mathcal{W}_{\mu}^{(m-k)} / \mathbb{P}_{m-k-1} \rightarrow \mathcal{W}_{\nu}^{(m+l-k-1)} / \mathbb{P}_{m+l-k-2}$, we get (45).

Now we prove that

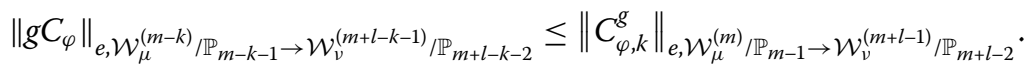

Since (31) holds for every $f \in \mathcal{W}_{\mu}^{(m-k)} / \mathbb{P}_{m-k-1}$ and by using (32), we see that the operator $I_{k}: \mathcal{W}_{\mu}^{(m-k)} / \mathbb{P}_{m-k-1} \rightarrow \mathcal{W}_{\mu}^{(m)} / \mathbb{P}_{m-1}$ is bounded. From this, since $C_{\varphi, k}^{g}: \mathcal{W}_{\mu}^{(m)} / \mathbb{P}_{m-1} \rightarrow$ $\mathcal{W}_{v}^{(m+l-1)} / \mathbb{P}_{m+l-2}$ is bounded by the assumption, and $D^{k}: \mathcal{W}_{v}^{(m+l-1)} / \mathbb{P}_{m+l-2} \rightarrow \mathcal{W}_{v}^{(m+l-k-1)} /$ $\mathbb{P}_{m+l-k-2}$ is also bounded due to the inequality in (34) and (46), we see that the operator $D^{k} C_{\varphi, k}^{g} I_{k}=g C_{\varphi}: \mathcal{W}_{\mu}^{(m-k)} / \mathbb{P}_{m-k-1} \rightarrow \mathcal{W}_{v}^{(m+l-k-1)} / \mathbb{P}_{m+l-k-2}$ is bounded. Beside this, 
note also that $C_{\varphi, k}^{g}=I_{k} g C_{\varphi} D^{k}: \mathcal{W}_{\mu}^{(m)} / \mathbb{P}_{m-1} \rightarrow \mathcal{W}_{v}^{(m+l-1)} / \mathbb{P}_{m+l-2}$, where $D^{k}: \mathcal{W}_{\mu}^{(m)} / \mathbb{P}_{m-1} \rightarrow$ $\mathcal{W}_{v}^{(m-k)} / \mathbb{P}_{m-k-1}$ and $I_{k}: \mathcal{W}_{\mu}^{(m+l-k-1)} / \mathbb{P}_{m+l-k-2} \rightarrow \mathcal{W}_{v}^{(m+l-1)} / \mathbb{P}_{m+l-2}$.

Let $K: \mathcal{W}_{\mu}^{(m)} / \mathbb{P}_{m-1} \rightarrow \mathcal{W}_{v}^{(m+l-1)} / \mathbb{P}_{m+l-2}$ be a compact operator. Then the operator

$$
D^{k} K I_{k}: \mathcal{W}_{\mu}^{(m-k)} / \mathbb{P}_{m-k-1} \rightarrow \mathcal{W}_{v}^{(m+l-k-1)} / \mathbb{P}_{m+l-k-2}
$$

is also compact.

Hence, as in (37) we have

$$
\left\|g C_{\varphi} f-D^{k} K I_{k} f\right\|_{\mathcal{W}_{\nu}^{(m+l-k-1)}} \leq\left\|C_{\varphi, k}^{g}-K\right\|_{\mathcal{W}_{\mu}^{(m)} / \mathbb{P}_{m-1} \rightarrow \mathcal{W}_{\nu}^{(m+l-1)} / \mathbb{P}_{m+l-2}}\|f\|_{\mathcal{W}_{\mu}^{(m-k)}} .
$$

By taking the supremum in (49) over the unit ball in $\mathcal{W}_{\mu}^{(m-k)} / \mathbb{P}_{m-k-1}$, and then taking the infimum in such obtained inequality over the set of all compact operators $K$ : $\mathcal{W}_{\mu}^{(m)} / \mathbb{P}_{m-1} \rightarrow \mathcal{W}_{v}^{(m+l-1)} / \mathbb{P}_{m+l-2}$, we get (48). From (45) and (48) is directly obtained (44), finishing the proof of the theorem.

\section{Competing interests}

The author declares that he has no competing interests.

\section{Authors' contributions}

The author has contributed solely to the writing of this paper. He read and approved the manuscript.

\section{Publisher's Note}

Springer Nature remains neutral with regard to jurisdictional claims in published maps and institutional affiliations.

Received: 25 June 2017 Accepted: 3 August 2017 Published online: 13 September 2017

\section{References}

1. Stević, S: Composition operators from the weighted Bergman space to the $n$-th weighted spaces on the unit disc. Discrete Dyn. Nat. Soc. 2009, Article ID 742019 (2009)

2. Stević, S: Composition operators from the Hardy space to the $n$th weighted-type space on the unit disk and the half-plane. Appl. Math. Comput. 215, 3950-3955 (2010)

3. Stević, S: Weighted differentiation composition operators from $H^{\infty}$ and Bloch spaces to $n$th weigthed-type spaces on the unit disk. Appl. Math. Comput. 216, 3634-3641 (2010)

4. Stević, S: Weighted differentiation composition operators from the mixed-norm space to the $n$th weigthed-type space on the unit disk. Abstr. Appl. Anal. 2010, Article ID 246287 (2010)

5. Bierstedt, KD, Summers, WH: Biduals of weighted Banach spaces of analytic functions. J. Aust. Math. Soc. Ser. A 54 70-79 (1993)

6. Stević, S: Essential norms of weighted composition operators from the $\alpha$-Bloch space to a weighted-type space on the unit ball. Abstr. Appl. Anal. 2008, Article ID 279691 (2008)

7. Avetisyan, KL: Hardy-Bloch type spaces and lacunary series on the polydisk. Glasg. Math. J. 49(2), 345-356 (2007)

8. Li, S, Stević, S: Volterra type operators on Zygmund space. J. Inequal. Appl. 2007, Article ID 32124 (2007)

9. Li, S, Stević, S: Generalized composition operators on Zygmund spaces and Bloch type spaces. J. Math. Anal. Appl. $338,1282-1295(2008)$

10. Stević, S: On an integral operator from the Zygmund space to the Bloch-type space on the unit ball. Glasg. Math. J. 51, 275-287 (2009)

11. Cowen, CC, MacCluer, BD: Composition Operators on Spaces of Analytic Functions. Studies in Advanced Mathematics. CRC Press, Boca Raton (1995)

12. Zhu, X: Weighted composition operators from logarithmic Bloch spaces to a class of weighted-type spaces in the unit ball. Ars Comb. 91, 87-95 (2009)

13. Li, S: On an integral-type operator from the Bloch space into the $Q_{K}(p, q)$ space. Filomat 26(2), 331-339 (2012)

14. Pan, C: On an integral-type operator from $Q_{K}(p, q)$ spaces to $\alpha$-Bloch spaces. Filomat 25(3), 163-173 (2011)

15. Stević, S, Sharma, AK: Integral-type operators from Bloch-type spaces to $Q_{K}$ spaces. Abstr. Appl. Anal. 2011, Article ID 698038 (2011)

16. Ueki, SI: On the Li-Stević integral type operators from weighted Bergman spaces into $\beta$-Zygmund spaces. Integral Equ. Oper. Theory 74(1), 137-150 (2012)

17. Zhu, X: On an integral-type operator between $H^{2}$ space and weighted Bergman spaces. Bull. Belg. Math. Soc. Simon Stevin 18(1), 63-71 (2011)

18. Stević, S: Products of integral-type operators and composition operators from the mixed norm space to Bloch-type spaces. Sib. Math. J. 50(4), 726-736 (2009)

19. Stević, S: On operator $P_{\varphi}^{9}$ from the logarithmic Bloch-type space to the mixed-norm space on unit ball. Appl. Math. Comput. 215, 4248-4255 (2010) 
20. Stević, S, Ueki, SI: Integral-type operators acting between weighted-type spaces on the unit ball. Appl. Math. Comput. 215, 2464-2471 (2009)

21. Yang, W, Yan, W: Generalized weighted composition operators from area Nevanlinna spaces to weighted-type spaces. Bull. Korean Math. Soc. 48(6), 1195-1205 (2011)

22. Yang, W, Zhu, X: Generalized weighted composition operators from area Nevanlinna spaces to Bloch-type spaces. Taiwan. J. Math. 16(3), 869-883 (2012)

23. Yu, Y, Liu, Y: On Stević type operator from $H^{\infty}$ space to the logarithmic Bloch spaces. Complex Anal. Oper. Theory 9(8), 1759-1780 (2015)

24. Zhu, X: Generalized weighted composition operators from Bloch-type spaces to weighted Bergman spaces. Indian J. Math. 49(2), 139-149 (2007)

25. Zhu, X: Multiplication followed by differentiation on Bloch-type spaces. Bull. Allahabad Math. Soc. 23(1), 25-39 (2008)

26. Zhu, X: Differentiation followed by composition from Bloch spaces to $Q_{p}$ spaces. J. Comput. Anal. Appl. 12(4), $731-738(2010)$

27. Zhu, X: Generalized weighted composition operators from Bloch spaces into Bers-type spaces. Filomat 26(6), 1163-1169 (2013)

28. Zhu, X: Generalized weighted composition operators on Bloch-type spaces. J. Inequal. Appl. 2015, Article ID 59 (2015)

29. Sanatpour, AH: Estimates of essential norms of generalized composition operators between Zygmund type spaces. Math. Inequal. Appl. 19(2), 685-696 (2016)

30. Stević, S: On a new integral-type operator from the Bloch space to Bloch-type spaces on the unit ball. J. Math. Anal. Appl. 354, 426-434 (2009)

31. Galindo, P, Lindström, M, Stević, S: Essential norm of operators into weighted-type spaces on the unit ball. Abstr. Appl. Anal. 2011, Article ID 939873 (2011)

\section{Submit your manuscript to a SpringerOpen ${ }^{\circ}$ journal and benefit from:}

- Convenient online submission

- Rigorous peer review

Open access: articles freely available online

- High visibility within the field

- Retaining the copyright to your article

Submit your next manuscript at $\boldsymbol{\nabla}$ springeropen.com 